\title{
腹水癌和白血病细胞从母体感染 胎儿频率的比较
}

颜永杉 冯尚 王永红 张 婷

(中国科学院遗传研究所, 北京 100101)

摘要 用小鼠动物模型证明腹水癌 Ehrlich 细胞可从母体进入胎儿体内, 成为继白血病 L615 细胞之后第二个可引起母子代癌感染的另一类型癌细胞. 在同一妊娠的多个胎儿中, 受母体 癌细胞感染的可能只有少数或个别的胎儿. 怀孕早期和中期为这两种癌细胞从母体感染胎儿 的危险期: 白血病 L615 和腹水癌 Ehrlich 细胞感染胎儿频率分别为 $100 \%$ 和 35\%; 但在怀孕 后期, 它们的感染频率分别降至 4. 5\% 和 0 . 可见, 白血病 L615 细胞引起母子代癌感染的频 率远远高于腹水癌 Ehrlich 细胞.

\section{关键词 家族性肿瘤 腹水癌和白血病 母体癌细胞 胎儿癌感染 标记染色体}

遗传性肿瘤( hereditary tumors) 或家族性肿瘤( family tumors) 一直是遗传学和临床医学 研究的重要课题. 近年来, 相继在人和其他哺乳类, 如猪、牛和兔的母体血液中发现有胎儿细 胞, 证明胎儿细胞可穿过胎盘进入母体 ${ }^{[1 ~ 3]}$. 文献 [4] 用一个小鼠白血病动物模型( L615), 首 次报道了母体白血病细胞可进入胎儿, 使胎儿患白血病. 本文报道用带标记染色体 Ehrlich 腹 水癌动物模型, 研究腹水癌细胞进入胎儿, 引起母子代之间腹水癌细胞感染的实验结果; 同时 还对腹水癌与白血病两种不同类型癌细胞感染胎儿的频率进行了比较. 国内外未见有关的报 道.

\section{1 材料与方法}

（）腹水癌细胞的体内传代和染色体分析. 将 Ehrlich 癌细胞注入健康昆明小鼠的腹 腔里, 7 $10 \mathrm{~d}$ 被接种的小鼠出现严重的腹水. 再将含 Ehrlich 癌细胞的腹水注入另外健康小 鼠, 进行体内传代. 按照文献 [4] 报道的方法制备癌细胞染色体片子和进行 C 带处理.

（）腹水癌细胞从母体进入胎儿体内实验. 往不同孕期的孕鼠腹腔注入 Ehrlich 癌细 胞 $\left(1 \times 10^{7}\right.$ 个癌细胞/ 只), 在 7 $12 \mathrm{~d}$ 后取出胎儿或待幼鼠出生, 再根据 Ehrlich 癌细胞的标记 染色体和染色体众数来检测胎儿或幼鼠体内是否有 Ehrlich 癌细胞. 由于进入胎儿体内的癌 细胞数量很少, 难以直接用来制备染色体片子, 因此我们采用在同品系小鼠体内扩大培养癌细 胞的方法, 即从每只孕鼠取出全部胎儿或将已出生的幼鼠用蒸馏水洗 10 15 次, 并在蒸馏水 中浸泡 $30 \mathrm{~min}$, 以除去胎儿表面可能存在的母体细胞, 然后用 $0.9 \% \mathrm{NaCl}$ 洗净, 最后把胎儿或 幼鼠剪碎, 制成细胞悬液, 注入多只健康昆明小鼠, 观察是否出现严重腹水. 若有严重腹水, 吸 出 $2 \mathrm{~mL}$ 腹水, 加到 RPM I1640(含 $10 \%$ 小牛血清和适量秋火仙素), $37{ }^{\circ} \mathrm{C}$ 培养 $2 \mathrm{~h}$, 然后制备染 色体片子, 进行染色体计数和 C 带分析. 为了进一步检测冲洗干净的胎儿体外是否还混有个 别母体血液中的癌细胞, 我们把最后 1 次冲洗胎儿的生理盐水, 注入健康昆明鼠体内, 定为对 照组. 只有当对照组为致癌阴性时, 其相应的实验组的数据才能被录用. 此外, 我们还对同一 
妊娠的每个胎儿分别进行致癌实验, 即把每个胎儿的细胞分别注入健康昆明小鼠并设有相应 对照组. 有 3 只孕鼠共 34 个胎儿被采用, 由于接种腹水癌细胞的小鼠 $5 \mathrm{~d}$ 后就会出现明显的 腹水, 并在接种后 17 $20 \mathrm{~d}$ 死亡, 因此如果实验组和对照组小鼠在两个半月后仍无大量腹水 出现, 而且解剖时未见瘤块者, 定为致癌阴性.

（）白血病细胞( L615) 从母体进入胎儿体内实验. 在文献 [4] 的基础上增加实验重复次 数. 以求得母体白血病细胞感染胎儿的感染频率 (表 1). 因每一个 L615 细胞都有 Bi 标记染色 体, 即一条 19 号染色体两端都为异染色质区 (C 带暗区), 很容易与宿主细胞区分开(图 1).

表 1 母鼠腹水癌 (Ehrlich) 和白血病 (L615) 细胞感染胎儿的频率

\begin{tabular}{|c|c|c|c|c|c|c|c|c|}
\hline \multirow[b]{2}{*}{ 癌细胞类型 } & \multirow{2}{*}{$\begin{array}{c}\text { 接种癌细胞 } \\
\text { 时母鼠 } \\
\text { 孕期/d } \\
\end{array}$} & \multicolumn{5}{|c|}{ 接种癌细胞的孕鼠数/只 } & \multicolumn{2}{|c|}{ 癌细胞感染率/\% } \\
\hline & & 总数 & $\begin{array}{c}\text { 出生幼鼠的 } \\
\text { 孕鼠数 }\end{array}$ & $\begin{array}{l}\text { 提供胎儿 } \\
\text { 的孕鼠数 }\end{array}$ & $\begin{array}{l}\text { 胎儿流产 } \\
\text { 的孥鼠数 }\end{array}$ & $\begin{array}{l}\text { 胎儿体内有癌 } \\
\text { 细胞的孕鼠数 }\end{array}$ & 实验组 & 对照组 \\
\hline 腹水癌 & $6 \sim 12$ & 30 & 0 & 20 & 10 & 7 & 35.0 & 0 \\
\hline Ehrlich & $14 \sim 17$ & 20 & 20 & 0 & 0 & 0 & 0 & 0 \\
\hline \multirow{2}{*}{ L615 } & $7 \sim 12$ & 35 & 0 & 35 & 0 & 35 & 100 & 0 \\
\hline & 15 & 22 & 22 & 0 & 0 & 1 & 4.5 & 0 \\
\hline
\end{tabular}

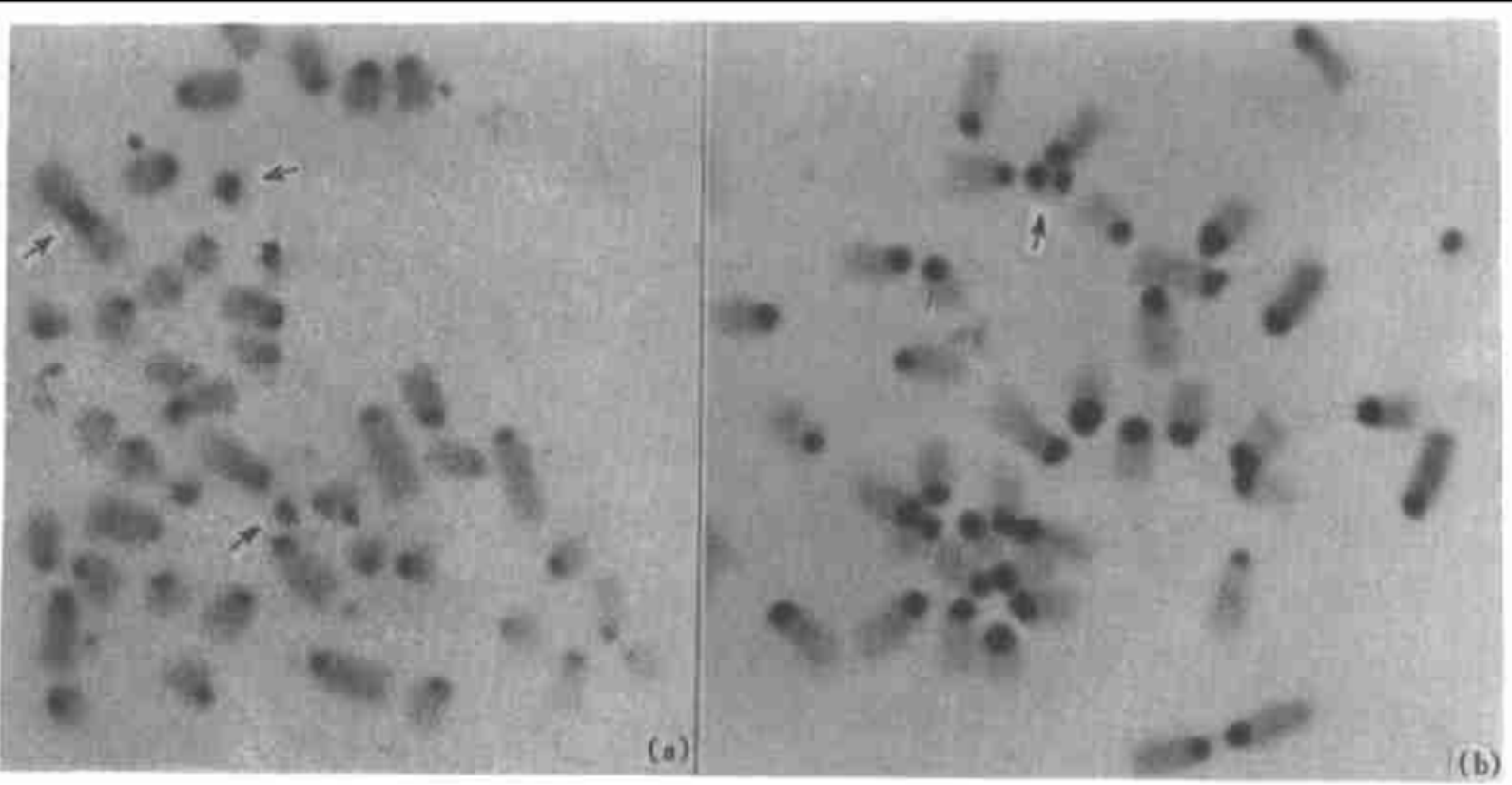

图 1 在小鼠胎儿体内发现来自母体的 Ehrlich 癌细胞( a) 和 L615 白血病细胞 (b) 的染色体图样 箭头所示为标记染色体

\section{2 结果}

\section{1 Ehrlich 癌细胞的染色体特征}

观察和统计 100 个 Ehrlich 癌细胞分裂相, 确定其染色体众数为 45, 并有很明显的标记染 色体: 1 条罗伯逊融合染色体和 2 条着丝粒有很大异染色质区的小染色体(图 1). 这些与宿主 小鼠染色体特征 ( $2 n=40$, 全为顶端着丝粒染色体) 明显不同, 因此可在显微镜下准确判断是 否有 Ehrlich 癌细胞.

2.2 母体 Ehrlich 腹水癌细胞感染胎儿

在怀孕早期和中期( 怀孕 6 12 d) 组合中, 共有 30 只孕鼠接种 Ehrlich 癌细胞. 其中 10 
只孕鼠在解剖后没有胎儿, 但在子宫壁上仍有胎盘着落点, 表明胎儿已流产, 无法进行胎儿致 癌实验; 余下 20 只孕鼠的胎儿发育正常. 在接种胎儿细胞后 5 $6 \mathrm{~d}, 20$ 只孕鼠中有 7 只孕鼠 的胎儿细胞引起实验组小鼠出现严重腹水. 染色体分析表明, 腹水的分裂相的染色体众数为 45, 并有 Ehrlich 细胞标记染色体, 为 Ehrlich 癌细胞. 其对照组为致癌阴性; 其他 13 只孕鼠的 胎儿全都为致癌阴性. 因此, Ehrlich 癌细胞在小鼠怀孕早期和中期使胎儿致癌的频率为 $35 \%$ (表 1). 但是. 在怀孕晚期( 怀孕 14 17 d) 组合中. 接种 Ehrlich 癌细胞的所有 20 只孕鼠的胎 儿都呈现致癌阴性. 表明在怀孕晚期, Ehrlich 癌细胞没有感染胎儿.

2.3 Ehrlich 癌细胞对同一妊娠的不同胎儿感染能力的差异

在 3 只孕鼠共 34 个胎儿的致癌实验中, 有 2 只孕鼠 (共 21 个胎儿) 的每个胎儿都呈现致 癌阴性; 在余下 1 只孕鼠所怀的 13 个胎儿中, 只有 1 个胎儿呈现致癌阳性, 而其对照组为致癌 阴性; 同一妊娠的其他 12 个胎儿却都呈现致癌阴性. 结果表明, 即使在接种 Ehrlich 癌细胞相 同的条件下, 不同胎儿受癌细胞感染的程度可有明显的不同.

\subsection{L615 白血病细胞感染胎儿的频率}

如表 1 所示, 在小鼠怀孕早期和中期( 怀孕 7 12 d) 接种白血病的 35 只孕鼠中, 在每只孕 鼠的胎儿体内都能找到带标记染色体 $(\mathrm{Bj})$ 的白血病细胞( 图 1), 表明每只孕鼠的胎儿都被白 血病细胞感染, 癌感染率为 $100 \%$; 在怀孕晚期接种白血病细胞的 22 只孕鼠中, 只有 1 只孕鼠 的胎儿被白血病感染, 癌感染率仅为 $4.5 \%$.

\section{3 讨论}

本文首次报道腹水癌细胞可从母鼠体内进入胎儿, 成为继白血病细胞 ${ }^{[4]}$ 之后能直接引起 母子代之间癌细胞感染的另一种类型癌细胞. 不论是白血病还是腹水癌, 哺乳类怀孕早期和 中期都是母体癌细胞感染胎儿的危险期. 其中, 白血病细胞引起母子代癌感染的频率远远高 于 Ehrlich 腹水癌细胞, 这可能与这两种类型癌细胞穿透血管壁引起癌转移的能力不同有关. 例如, L615 白血病细胞非常容易转移至肺和脑部形成病灶. 本文还证明了在同一妊娠的多个 胎儿中, 并非所有的胎儿都同样受到母体癌细胞的感染, 受母体癌细胞感染的可能只有个别的 很少数的胎儿. 癌细胞很可能以穿透或侵袭等方式从母体进入胎儿, 引起母子代癌感染. 本 文结果对人类的家族性癌传递机制研究有重要的参考价值.

致谢 本工作为国家自然科学基金(批准号: 39770372) 和中国科学院重点基金( 批准号: 6_153410) 资助项目.

\section{参 考 文 献}

1 Herzenberg L A, Bianchi D W, Schroder J, et al. Fetal cells in the blood of proegnant woman: detection and enrichment by fluorescence_activated cell sorting. Proc Natl Acad Sci USA, 1979, 76: 1 453 1455

2 Rudek Z, Kw iatkow ska L. The possibility of detecting fefal lymphocytes in the maternal blood of the demestic pig. Cytogenet Cell Genet, 1983, 36: 580 583

3 Bianchi D W, Mahr A, Zickwolf G K, et al. Detection of fetal cells with 47, XY, + 21 Karyotype in maternal perpheral blood. Hum Genet, 1992, 90: 368 370

4 吴晓辉, 颜永杉. 哺乳类胎盘的细胞通透性及其遗传学意义. 科学通报, 1993, 38(20)：1900 1902

(1998-01-08 收稿, 1998-04-03 收修改稿) 